\title{
Targeted, Monitored, and Controlled Chemotherapy: A Multimodal Nanotechnology-Based Approach against Cancer
}

\author{
Ali Shakeri-Zadeh, ${ }^{1}$ Samideh Khoei, ${ }^{1}$ Sepideh Khoee, \\ Ali Mohammad Sharifi, ${ }^{3}$ and Mohammad-Bagher Shiran ${ }^{1}$ \\ ${ }^{1}$ Department of Medical Physics, Faculty of Medicine, Tehran University of Medical Sciences (TUMS), Tehran 14155-5983, Iran \\ ${ }^{2}$ School of Chemistry, University College of Science, University of Tehran, Tehran, Iran \\ ${ }^{3}$ Razi Drug Research Center and Department of Pharmacology, School of Medicine, Tehran University of Medical Sciences (TUMS), \\ Tehran, Iran
}

Correspondence should be addressed to Mohammad-Bagher Shiran; mshiran@tums.ac.ir

Received 10 May 2013; Accepted 19 July 2013

Academic Editors: A. Hu and A. Taubert

Copyright (C) 2013 Ali Shakeri-Zadeh et al. This is an open access article distributed under the Creative Commons Attribution License, which permits unrestricted use, distribution, and reproduction in any medium, provided the original work is properly cited.

\begin{abstract}
We review innovative methods for treatment of cancer tumor on the basis of nanotechnology and physics to target, monitor and control release of chemotherapeutic agents. Chemotherapy is one of the main methods of treatment for cancer and plays a vital role in clinical practice, but side effects of anticancer drugs are still critical problems. Magnetic nanoparticles can be applied as an effective drug carriers and contrast agents for magnetic resonance imaging (MRI). Since certain nanoparticles have magnetic properties, they can be trapped in tumor during blood circulation by an external magnetic field. Also, polymeric nanoparticles are great candidates to encapsulate anticancer drugs and to control the release profile of drugs in biologic media. We suggest the construction of drug-loaded polymer-coated magnetic (DPM) nanoplatform with the potential for being utilized in medical imaging as well as having controlled drug release properties. Nanoplatform distribution can be monitored by MRI and with clever combination of ultrasound physics and suggested DPM nanoplatform, it would be feasible to increase the rate of drug release (in situ) and drug uptake by cancerous cell. To optimize the level of drug uptake by cancerous cell, the selection of ultrasound frequency and intensity is essential. The development of suggested method could be a new approach against cancer tumor.
\end{abstract}

\section{Introduction}

1.1. Cancer. Cancer is a disease of the cell in which the normal mechanisms of cell growth and proliferation are disturbed. Among all treatments for cancer (surgery, chemotherapy, and radiotherapy), chemotherapy plays an important role in clinical practice, but the drugs' side effects are the main problems $[1,2]$. Anticancer agents are not specific to just targeting cancer cells and always lead to systemic toxicity. As a result, these drugs cause undesirable and severe side effects such as hair loss, renal and hepatic damages, and bone marrow [3]. Therefore, one of the major disadvantages of the chemotherapy is the destruction of cancer cells as well as normal cells of the body. This difficulty would be minimized if we concentrate the drug in the tumor region which in turn leads to the increase of antitumor efficacy with applying a locally higher but systemically lower dose.
Also from economical point of view, administrating a lower dose drugs systematically can be taken into consideration. Accordingly, an exciting potential solution for this problem is to encapsulate the drug in biocompatible and biodegradable materials that can be injected into the blood stream with the intention of delivering the drug to the effected region.

1.2. Magnetic Nanoparticles. Magnetic nanoparticles are an important class of functional materials which have attracted the attention of many researchers and have many potential applications in the area of bioscience and medicine. Because of having superparamagnetic properties, the main applications of magnetic nanoparticles are as follows $[4,5]$ :

(i) effective agents for thermotherapy (hyperthermia) because of their hysteresis loss in the alternative magnetic field; 
(ii) contrast agent for magnetic resonance imaging (MRI);

(iii) good carriers for drug delivery.

1.3. Polymeric Nanoparticles. The use of biodegradable polymeric nanoparticle as a drug delivery system offers various important advantages as follows: (i) accurately controlling the release rate of an incorporated drug, (ii) easy administration of the drug, and (iii) no need for (surgical) removal. For example, poly(lactic-co-glycolic acid) (PLGA) is one of the most important polymers which is frequently used for the encapsulation of anti-cancer agents [6]. Due to their intelligent behavior in response to physicochemical variations, polymers have gained an increasing attention in the field of controlled drug release. Passive targeting or enhanced penetration and retention (EPR) effect is the most important advantage of polymeric nanoparticles that provides such nanoparticles for a selective accumulation in solid tumors. It is worthy to note that when a drug is encapsulated in polymeric nanoparticles, its intracellular uptake by all types of cells is substantially reduced. This way, unwanted drug interactions with normal cells can be prevented.

There still exists an important problem, and it is the requirement for a technique to provide the effective intracellular drug uptake at a tumor site. In order to resolve this problem, we recommend the use of magnetic force and ultrasound which will be discussed in the following.

1.4. Magnetic Drug Targeting and Monitoring. A need exists for anti-cancer drugs to specifically target the tumor site, without damaging normal body cells [1]. This process is called "drug targeting," that is, directing a drug as precisely as possible to the desired region, effectively mitigates harmful side effects and offers the possibility to apply a systemically lower dose [7].

Over the last 20 years, different approaches to drug targeting of chemotherapeutics have been pursued. Magnetic drug targeting (MDT) is one the most important types of drug targeting methods in which a drug is accompanied by a magnetic substance (ferrofluid) and directed into the desired region by an externally applied magnet [8]. The concept of MDT is not new [9], and the goal of this strategy is the concentration of drug at a target site, far away from the reticular endothelial system, with the drive of a magnetic field.

Polymeric and magnetic nanoparticles can be combined to formulate a new type of nanoplatform. For instance, the use of magnetic iron oxide in polymer matrices (such as PLGA) has been recently reported [10] to impart a magnetic moment to the polymeric nanoparticles. Since these nanoplatforms have a magnetic core, the accumulation of anti-cancer drug in specific location can be monitored by MRI.

1.5. Ultrasound and Drug Delivery. Ultrasound is being used for enhancement of drug uptake in vitro and in vivo [11]. Recently, it has been demonstrated that the interaction of ultrasound with nanoparticles may enhance drug delivery in tumor cells in vivo which is due to alteration of tumor vasculature and the cell membrane. The contribution of lowintensity ultrasound in enhancing the drug in the tissue is due to mechanical membrane stretching of cell and nanoparticles, radiation force, and acoustic streaming [12]. The most important advantage of ultrasound in drug delivery application is that it is noninvasive, can penetrate through the body, and can be carefully controlled via a number of parameters including frequency, power density, duty cycles, and time of application [13].

Combination of ultrasound and polymeric nanoparticles provides us with passive targeting to the tumor volume in vivo and substantially enhances the intracellular drug uptake by tumor cells. Other studies show the anticancer agents that are loaded in polymeric nanoparticles and offer a possible solution for the improvement of tumor-therapeutic efficacy [14]. Upon the administration of polymeric nanoparticles into the biological system, an effective drug uptake by tumors should take place $[15,16]$.

Therefore, we suggest the construction of drug-loaded polymer-coated magnetic (DPM) nanoplatform with the potential for being utilized in medical imaging as well as possessing controlled drug release properties. Nanoplatform distribution can also be monitored by MRI, permitting quantification of drug delivery in real time in vivo. On the other hand, with clever combination of ultrasound and suggested DPM nanoplatform, it would be feasible to monitor and increase the drug release rate (in situ) and drug uptake by tumor $[15,16]$.

\section{Targeting Tumor with New Nanoplatforms and Ultrasound}

As mentioned in the Introduction, magnetic and polymeric nanoparticles loaded with a specific anticancer agent can be used for simultaneously drug targeting, monitoring, and release. Here, the construction of suggested nanoplatform is to be recognized as DPM nanoplatform and is composed of three distinct components. Firstly, a bio-degradable and biocompatible polymer is used as a matrix for loading and subsequent controlled release of therapeutic agents into cells. Secondly, magnetic nanoparticles are incorporated into the polymeric matrix which will be employed for both magnetically guided delivery (magnetic drug targeting) and MRI contrast agent (drug monitoring). Thirdly, an effective anti-cancer drug is used as a therapeutic agent for cancer. Accordingly, with clever combination of ultrasound and such a new nanotechnology platform, a multimodal approach against cancer is proposed and should be able to

(i) take the drug to a specific site of human the body using an external magnetic field (magnetic drug targeting),

(ii) to be able to monitor the drug using MRI (since the nanoplatform has magnetic core),

(iii) guarantee a well-defined drug release (due to polymer properties),

(iv) increase the release rate of drug from polymeric matrix by ultrasound exposure to the tumor, 


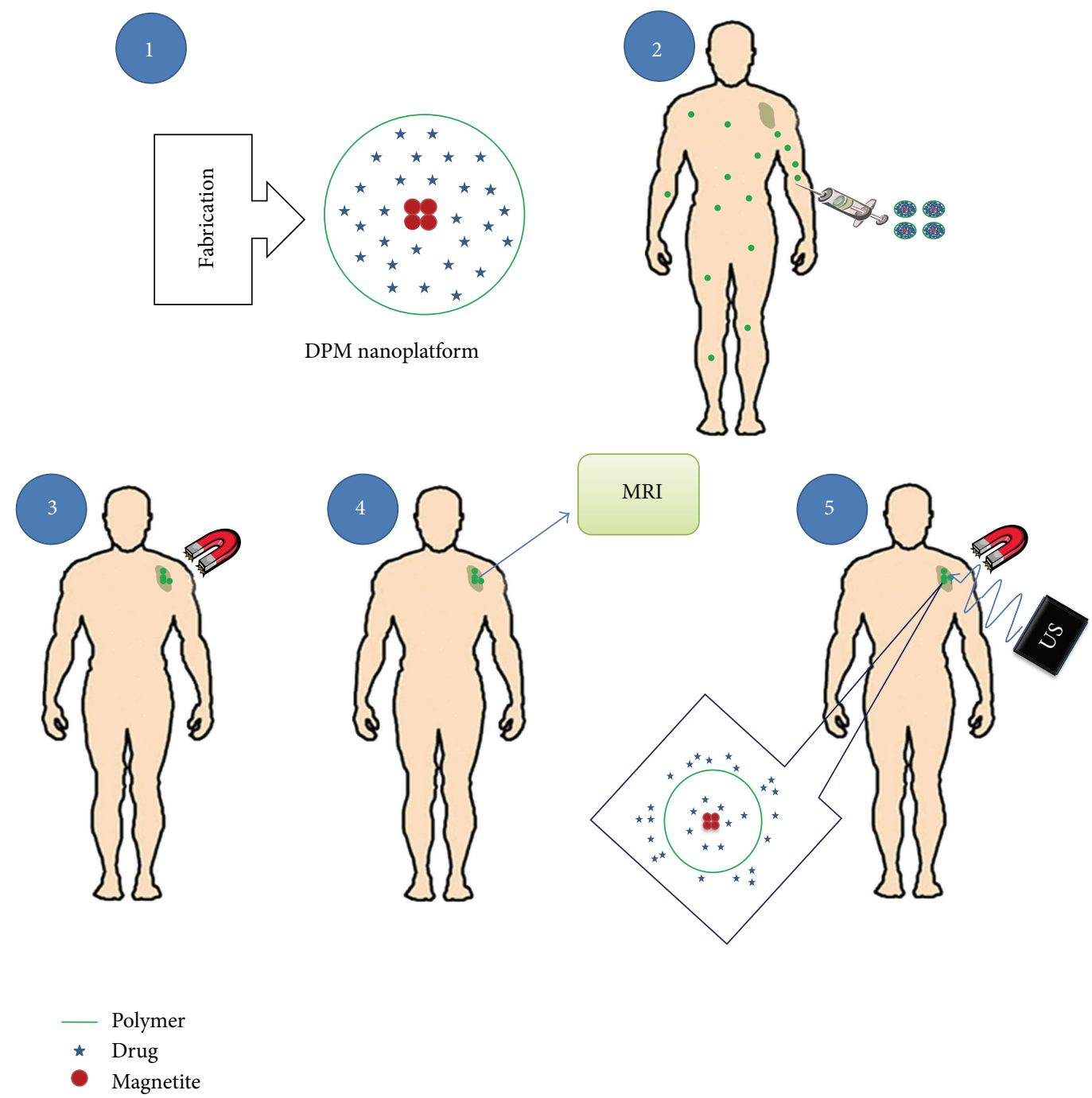

FIGURE 1: Schematic of presented multimodal approach for cancer therapy.

(v) increase drug uptake by cancer cells through sonication.

This approach enables a multimodal therapy that may provide efficient treatment against cancer. Figure 1 illustrates a schematic sequence of our idea step-by-step.

This multimodal treatment concept has to be converted into a practical patient treatment system with the following procedure:

(i) construction of the suggested (DPM) nanoplatform [1],

(ii) administration of DPM nanoplatform [2],

(iii) applying an appropriate external magnetic field accumulating DPM nanoplatforms in the tumor [3],

(iv) monitoring the accumulated DPM nanoplatforms by MRI [4],

(v) final phase, sonication of tumor impregnated by DPM nanoplatforms [5].

\section{Feasibility Study of Nanoplatform Construction}

We proposed a new modality of drug targeting to tumors based on the encapsulation of drugs in polymeric-magnetic nanoplatforms followed by a localized triggering of intracellular uptake of the drug induced by ultrasound focused in the tumor volume. The logic behind this approach is that drug encapsulation in the suggested nanoplatform decreases the systemic concentration of free drug, diminishes intracellular drug uptake by normal cells, and provides a passive drug targeting to tumors via EPR effect. Based on other studies, we discuss how to make the proposed idea become a reality.

To fabricate DPM nanoplatforms, suitable biodegradable polymers must be chosen. Natural or synthetic polymers are incorporated into magnetic nanoparticles and drugs using various techniques. Poly(lactide-co-glycolide) (PLGA) produces biocompatible and nontoxic subproducts that are further eliminated by normal metabolic pathways. This polymer has been approved by the Food and Drug Administration 
for certain clinical uses and has been utilized widely in drug delivery systems as an effective encapsulating material. Recently, we have used PLGA, which is a biodegradable copolymer, as a biomedical material and drug carrier in controlled release delivery systems [10]. In our study, magnetite nanoparticles and 5-fluorouracil (5-Fu) were incorporated into PLGA nanocapsules. This study suggests a prospective technique to prepare biodegradable DPM nanoplatform containing water-soluble agents such as 5 -Fu.

Through the magnetic drug targeting process, DPM nanoplatforms can be trapped by an external magnetic field during blood circulation in tumor. Alexiou et al. have many publications on fabrication of a specific nanoplatform which is accompanied by mitoxantrone as an anti-cancer agent and have successfully demonstrated that magnetic drug targeting can enhance the efficacy of chemotherapy $[7,17,18]$.

In the context of magnetic drug targeting and monitoring, after applying magnetic drug targeting, Lemke et al. [8] evaluated the ability of MRI to detect magnetic particle uptake into advanced solid malignant tumors of patients and documented the extension of these tumors. They concluded that MRI is a suitable method to detect magnetite particles, deposited at the tumor site via magnetic drug targeting.

Recently, Schroeder et al. [19] have presented a modality for the release of drugs from liposomes in vivo using ultrasound and have studied the ability of sonication to (a) trigger the release of cisplatin (an anti-cancer chemotherapeutic agent) from nanoliposomes in vivo and (b) enhance the therapeutic efficacy by locally releasing the drug. They have tested the effect of ultrasound-induced drug release on the therapeutic efficacy on $\mathrm{BALB} / \mathrm{c}$ mice with $\mathrm{C} 26$ colon adenocarcinoma tumors. They have reported that nearly $70 \%$ of the liposomal cisplatin was released in those tumors that are exposed to ultrasound, while the release was about 3\% in those that were not exposed to ultrasound. Interestingly, the group treated by liposomal cisplatin combined with ultrasound, compared to all other groups (i.e., free cisplatin with or without sonication, or liposomal cisplatin without sonication, or ultrasound alone, or no treatment), have had the best therapeutic results.

In another study, Kruskal et al. [20] have confirmed a paradigm of tumor targeting via drug delivery in a carrier (liposome-encapsulated doxorubicin) followed by ultrasonic irradiation of the tumor. In their experiments, a human liver tumor model in mice has been used and the carrier was injected systemically. Then, a burst of ultrasound was directed to the tumor 90 minutes after the drug injection. Their results show that the concentration of doxorubicin in sonicated tumors is doubled compared to nonsonicated tumors. Moreover, effective enhancement of vascular permeability for nanoparticle delivery into the sonicated tumors has been corroborated by Lin et al. [16] and their study indicates that sonication of tumor is promising for a better nanoparticle delivery in nanotechnology-based chemotherapy.

According to the aforementioned evidence, it seems that our idea has the potential for treatment of the localized cancers such as prostate or liver, by fixing a new multimodal approach using the concepts of both nanotechnology and ultrasound physics. In practice, the proposed idea in this paper can be tested for cancers (e.g., prostate cancer) in this way as follows:

(i) Fabrication of a new DPM nanoplatform composed magnetite (as magnetic core), 5-Fu (as a drug), and PLGA (as polymeric matrix).

(ii) Injection of DPM nanoplatform to different groups of xenograft model of human prostate carcinoma in nude mice. DU145 is a suitable candidate to be selected as a human prostate cell line.

(iii) Concentration of DPM nanoplatform in tumor through MDT process.

(iv) Taking MR images and making sure of the presence of DPM nanoplatforms in tumor.

(v) Sonication of tumor.

(vi) Carrying out various cellular and molecular tests to evaluate the effects of the suggested approach.

\section{Conclusion}

Toxic side effects of the anti-cancer drugs are the most important complications of chemotherapy. Here, we proposed a technique that holds promise in overcoming those complications. The technique is based on loading anticancer drug on polymeric-magnetic nanoplatforms and magnetically targeting them to the tumor followed by controlled release at the tumor site triggered by localized sonication. Additional modifications of the nanoplatform with monoclonal antibodies, folic acid, lectins, peptides, or hormones make the suggested technique more efficient and also highly specific [1, 21-25].

In conclusion, the realization of presented idea is indispensable and possible. In our laboratory we are developing a new modality of magnetic drug targeting to prostate tumor based on the drug loading on polymeric-magnetic nanoplatforms followed by a controlled release at the tumor site by sonication.

\section{Conflict of Interests}

The authors declared that there is no conflict of interests.

\section{Acknowledgments}

This work was supported by Grant no. 15026 from the Faculty of Medicine of Tehran University of Medical Sciences (TUMS).

\section{References}

[1] G. Ali Mansoori, K. S. Brandenburg, and A. Shakeri-Zadeh, "A comparative study of two folate-conjugated gold nanoparticles for cancer nanotechnology applications," Cancers, vol. 2, no. 4, pp. 1911-1928, 2010.

[2] Y. Zhang, B. Wang, Y. Wang, W. Qiao, and P. Shao, "A novel hypurgia for cancer chemotherapy: programmable release of 
antineoplastics and cytothesis agents from core-shell micro/nano-particles," Medical Hypotheses, vol. 76, no. 2, pp. 201-203, 2011.

[3] J. R. Senior, "Unintended hepatic adverse events associated with cancer chemotherapy," Toxicologic Pathology, vol. 38, no. 1, pp. 142-147, 2010.

[4] G. Ciofani, C. Riggio, V. Raffa, A. Menciassi, and A. Cuschieri, "A bi-modal approach against cancer: magnetic alginate nanoparticles for combined chemotherapy and hyperthermia," Medical Hypotheses, vol. 73, no. 1, pp. 80-82, 2009.

[5] A. Jordan, P. Wust, H. Fahling, W. John, A. Hinz, and R. Felix, "Inductive heating of ferrimagnetic particles and magnetic fluids: physical evaluation of their potential for hyperthermia," International Journal of Hyperthermia, vol. 9, no. 1, pp. 51-68, 1993.

[6] J. Siepmann, N. Faisant, J. Akiki, J. Richard, and J. P. Benoit, "Effect of the size of biodegradable microparticles on drug release: experiment and theory," Journal of Controlled Release, vol. 96, no. 1, pp. 123-134, 2004.

[7] C. Alexiou, R. Jurgons, R. J. Schmid et al., "Magnetic drug targeting-biodistribution of the magnetic carrier and the chemotherapeutic agent mitoxantrone after locoregional cancer treatment," Journal of Drug Targeting, vol. 11, no. 3, pp. 139-149, 2003.

[8] A.-J. Lemke, M.-I. Senfft von Pilsach, A. Lübbe, C. Bergemann, H. Riess, and R. Felix, "MRI after magnetic drug targeting in patients with advanced solid malignant tumors," European Radiology, vol. 14, no. 11, pp. 1949-1955, 2004.

[9] K. Mosbach and U. Schroder, "Preparation and application of magnetic polymers for targeting of drugs," FEBS Letters, vol. 102, no. 1, pp. 112-116, 1979.

[10] M. Ashjari, S. Khoee, and A. R. Mahdavian, "A multiple emulsion method for loading 5-fluorouracil into a magnetiteloaded nanocapsule: a physicochemical investigation," Polymer International, vol. 61, no. 5, pp. 850-859, 2012.

[11] M. B. Shiran, M. Motevalian, R. Ravanfar, and S. Bohlooli, “The effect of bubble surface charge on phonophoresis: implication in transdermal piroxicam delivery," Iranian Journal of Pharmacology and Therapeutics, vol. 7, no. 1, pp. 15-19, 2008.

[12] I. V. Larina, B. M. Evers, T. V. Ashitkov, C. Bartels, K. V. Larin, and R. O. Esenaliev, "Enhancement of drug delivery in tumors by using interaction of nanoparticles with ultrasound radiation," Technology in Cancer Research and Treatment, vol. 4, no. 2, pp. 217-226, 2005.

[13] N. Y. Rapoport, D. A. Christensen, H. D. Fain, L. Barrows, and Z. Gao, "Ultrasound-triggered drug targeting of tumors in vitro and in vivo," Ultrasonics, vol. 42, no. 1-9, pp. 943-950, 2004.

[14] Y. Liu, H. Miyoshi, and M. Nakamura, "Nanomedicine for drug delivery and imaging: a promising avenue for cancer therapy and diagnosis using targeted functional nanoparticles," International Journal of Cancer, vol. 120, no. 12, pp. 2527-2537, 2007.

[15] N. Y. Rapoport, A. M. Kennedy, J. E. Shea, C. L. Scaife, and K.-H. Nam, "Controlled and targeted tumor chemotherapy by ultrasound-activated nanoemulsions/microbubbles," Journal of Controlled Release, vol. 138, no. 3, pp. 268-276, 2009.

[16] C.-Y. Lin, Y.-L. Huang, J.-R. Li, F.-H. Chang, and W.-L. Lin, "Effects of focused ultrasound and microbubbles on the vascular permeability of nanoparticles delivered into mouse tumors," Ultrasound in Medicine and Biology, vol. 36, no. 9, pp. 14601469, 2010.
[17] A. S. Lübbe, C. Alexiou, and C. Bergemann, "Clinical applications of magnetic drug targeting," Journal of Surgical Research, vol. 95, no. 2, pp. 200-206, 2001.

[18] C. Alexiou, W. Arnold, R. J. Klein et al., "Locoregional cancer treatment with magnetic drug targeting," Cancer Research, vol. 60, no. 23, pp. 6641-6648, 2000.

[19] A. Schroeder, R. Honen, K. Turjeman, A. Gabizon, J. Kost, and Y. Barenholz, "Ultrasound triggered release of cisplatin from liposomes in murine tumors," Journal of Controlled Release, vol. 137, no. 1, pp. 63-68, 2009.

[20] J. Kruskal, S. Goldberg, and R. Kane, Eds., Novel In Vivo Use of Conventional Ultrasound to Guide and Enhance Molecular Delivery and Uptake into Solid Tumors, 2001.

[21] A. Shakeri-Zadeh, G. A. Mansoori, A. R. Hashemian, H. Eshghi, A. Sazgarnia, and A. R. Montazerabadi, "Cancerous cells targeting and destruction using folate conjugated gold nanoparticles," Dynamic Biochemistry, Process Biotechnology and Molecular Biology, vol. 4, pp. 6-12, 2010.

[22] A. Hashemian, H. Eshghi, G. Mansoori, A. Shakeri-Zadeh, and A. Mehdizadeh, "Folate-conjugated gold nanoparticles (synthesis, characterization and design for cancer cells nanotechnology-based targeting)," International Journal of Nanoscience and Nanotechnology, vol. 5, no. 1, article 25, 2010.

[23] A. Shakeri-Zadeh, M. Ghasemifard, and G. Ali Mansoori, "Structural and optical characterization of folate-conjugated gold-nanoparticles," Physica E: Low-Dimensional Systems and Nanostructures, vol. 42, no. 5, pp. 1272-1280, 2010.

[24] A. Shakeri-Zadeh, H. Eshghi, G. A. Mansoori, and A. R. Hashemian, "Gold nanoparticles conjugated with folic acid using mercaptohexanol as the linker," Journal Nanotechnology Progress International, vol. 1, pp. 13-23, 2009.

[25] K. S. Brandenburg, A. Shakeri-Zadeh, R. Hashemian, and G. A. Mansoori, "Folate-conjugated gold nanoparticles for cancer nanotechnology applications," in Proceedings of Nanotechnology Conference, pp. 404-407, June 2011. 

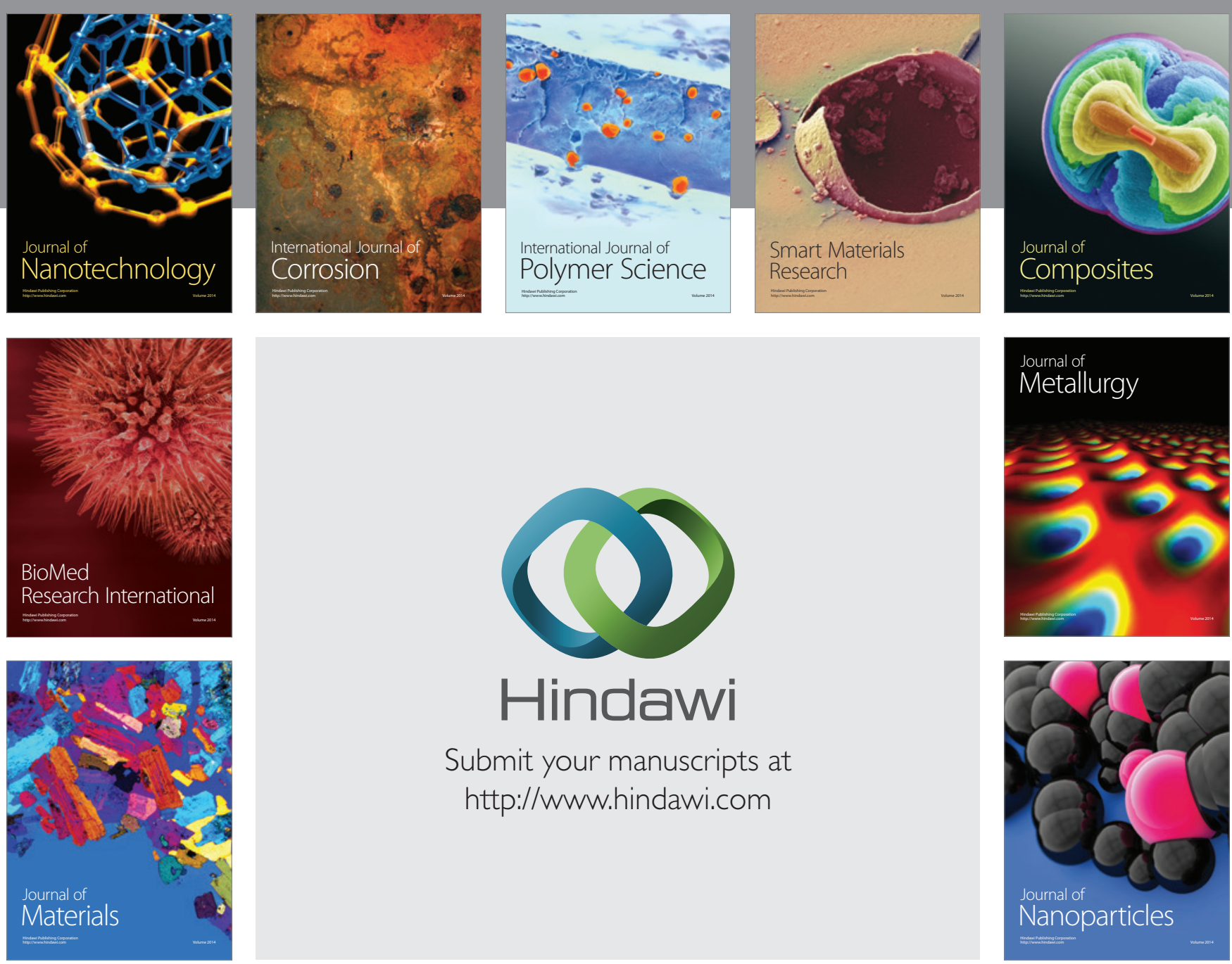

Submit your manuscripts at http://www.hindawi.com
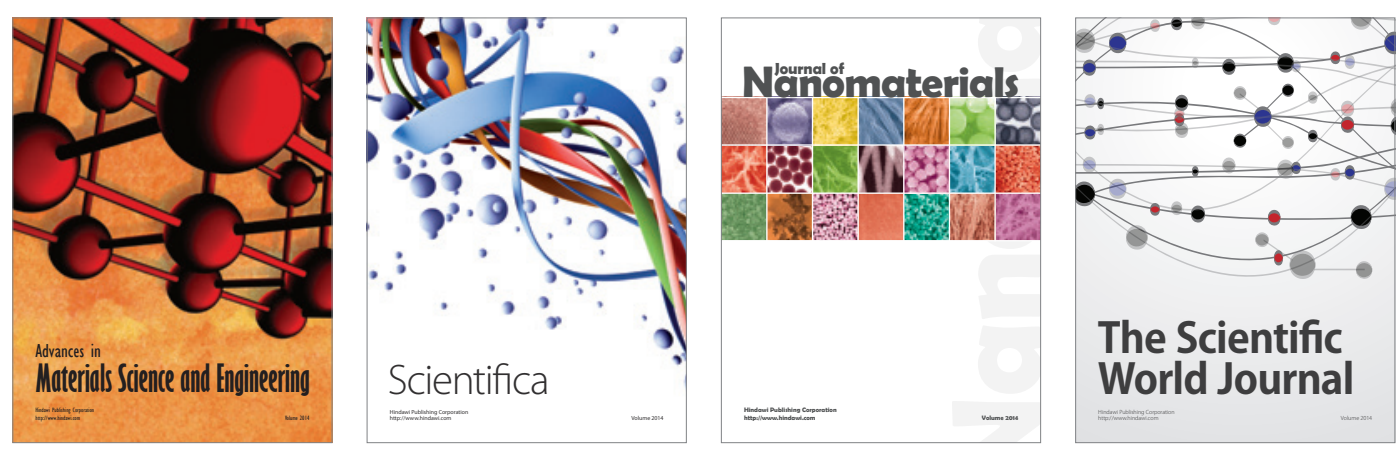

\section{The Scientific World Journal}
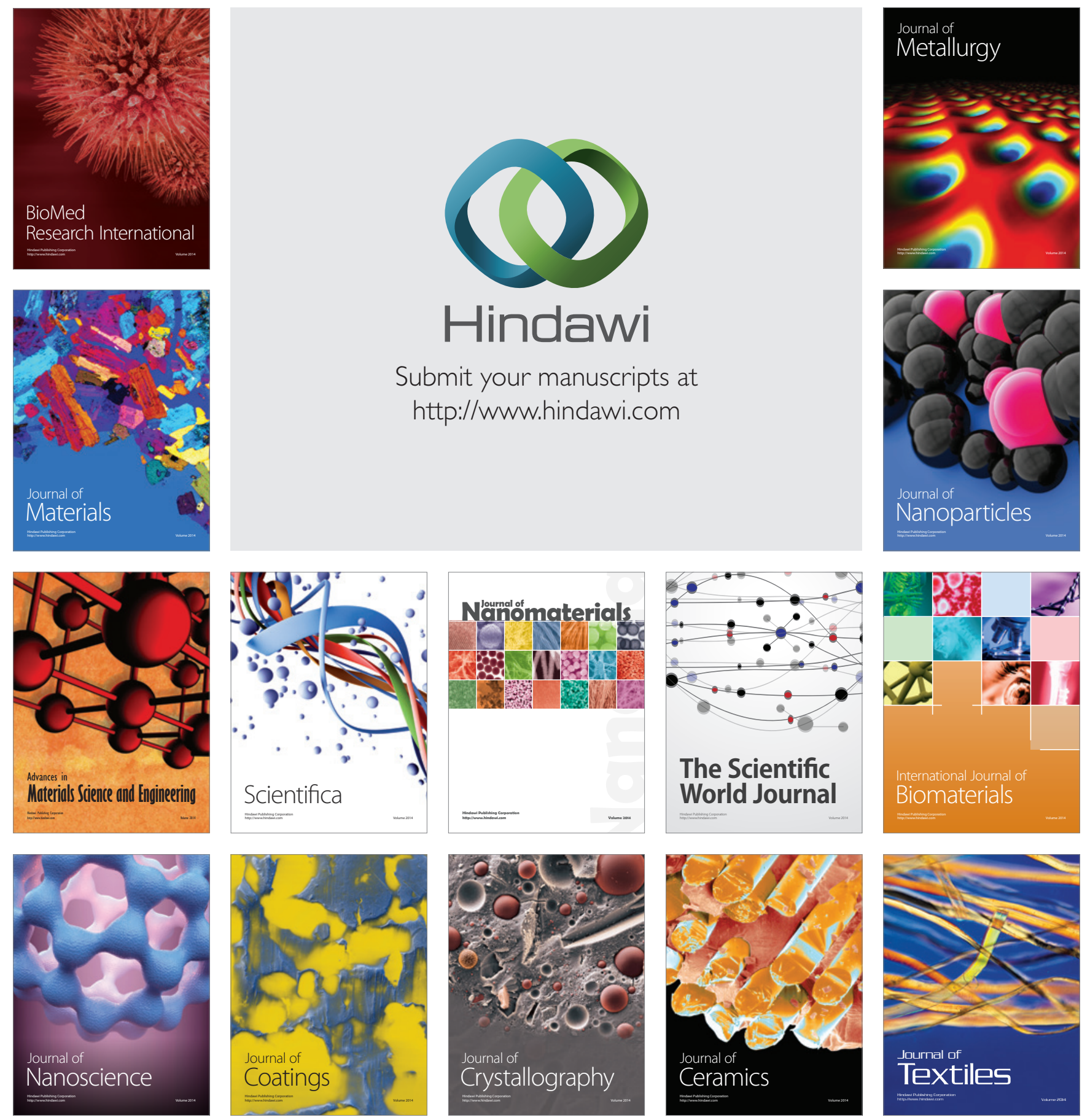